\title{
Una ciencia para la construcción de un mundo mejor. Aproximaciones a la Investigación para la $\mathbf{P a z}^{2}$
}

\author{
A science for the construction of a better world. \\ Approaches to research towards peace
}

\begin{abstract}
Resumen
Este es un artículo de revisión que presenta una mirada sucinta a esa disciplina científica denominada Investigación para la Paz. El trabajo realiza una aproximación a dicha disciplina a partir de las características que la componen y del análisis de su evolución histórica desde sus inicios, a comienzos del siglo $\mathrm{XX}$, hasta la actualidad. Para terminar, este estudio muestra las áreas de trabajo de la Investigación para la Paz y las tendencias actuales que la guían.
\end{abstract}

Palabras clave: Investigación para la Paz, resolución de conflictos, conflictividad internacional.

\begin{abstract}
This review article presents a brief look at the scientific discipline called Peace Research. The work is an approach to the discipline. First, there is an examination of its component characteristics. Second, there is an analysis of its historical evolution since its inception in the early twentieth century to nowadays. Finally, this study shows the work areas of the Peace Research and those trends that guide it.
\end{abstract}

Keywords: Peace Research, Conflict Resolution, International Conflicts.

Recibido el 6 julio de 2012 y aprobado el 27 septiembre de 2012

1 Doctor en Historia Contemporánea, Universidad de Granada. Trabaja en el Centro de Estudios para la Paz y la Reconciliación de la Universidad de Coventry, Reino Unido. Correos electrónicos: diegoch@ugr.es diego.checahidalgo@coventry.ac.uk

2 Este trabajo ha sido realizado con el apoyo del programa Marie Curie Intra European Fellowship, del 7 Programa Marco de la Comunidad Europea. 


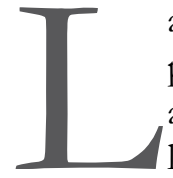

a Investigación para la Paz es un campo interdisciplinar que comprende el análisis sistemático de las causas de la violencia y las condiciones para la paz. Su origen se sitúa en la primera mitad del siglo XX, producto tanto de la reacción a las dos contiendas que asolaron a la humanidad, la Primera y la Segunda Guerra Mundial, como del continuo progreso de las Ciencias Sociales, que favoreció la aparición de grupos de científicos e investigadores que creían necesario abordar tales problemáticas con el máximo rigor y con la mayor parte de los recursos intelectuales disponibles. Con el paso del tiempo, la disciplina se fue definiendo a partir del estudio de tres ejes fundamentales: la paz, la violencia y los conflictos, y de las relaciones existentes entre ellos. En este sentido, los enfoques e interpretaciones propuestos desde la Investigación para la $\mathrm{Paz}$ resultan fundamentales para el análisis de muchas de las problemáticas presentes en el seno de las dinámicas de las sociedades y de las relaciones internacionales contemporáneas.

\section{Características de la Investigación para la Paz}

Cuando hablamos de Investigación para la Paz nos referimos a un campo de estudio definido cuya naturaleza y desarrollo se ha ido construyendo sobre una serie de elementos que, para los profesores Paul Rogers y Oliver Ramsbotham, pueden ser concretados en siete: la preocupación tanto por abordar la violencia en sus distintas manifestaciones y promover la paz; la necesidad de ofrecer una respuesta interdisciplinaria, dada la naturaleza multifacética del conflicto violento; la apuesta por la gestión pacífica y no violenta de los conflictos; la adhesión a un análisis multinivel de los conflictos que integrara las dimensiones individual, grupal, estatal e interestatal; la adopción de un enfoque global y multicultural; la conjunción de tareas analíticas con un compromiso normativo; y la estrecha relación entre teoría y práctica (Rogers \& Ramsbotham, 1999). A continuación vamos a abordar con más detenimiento cada uno de los elementos mencionados.

\section{A. Las preocupaciones por abordar la violencia y promover la paz}

A lo largo de su existencia, la Investigación para la Paz ha perseguido una agenda en la que ha tenido cabida tanto la preocupación por abordar las causas de la violencia directa como por explorar los caminos que permitan superar las desigualdades estructurales y promover unas relaciones equitativas y cooperativas.

Si bien su interés estaba inicialmente centrado en las circunstancias directamente relacionadas con la violencia directa y con los aspectos bélicos (dinámicas de las guerras, armas, violencia, agresión, etc.), paulatinamente se fueron incorporando nuevos temas como la educación para la paz, la resolución de conflictos, los pro- 
cesos de negociación y mediación, la cooperación y el desarrollo, los conflictos ambientales, la interculturalidad, la violencia de género, la globalización, los conflictos sociales prolongados, etc. (Jeong, 1999).

En este proceso se pudo distinguir una clara polarización dentro de la disciplina, entre aquellos minimalistas, como Kenneth Boulding, que definen la paz como la ausencia de violencia directa (paz negativa) (1977, pp. 77-86), y los maximalistas, como Johan Galtung, que añaden a ese concepto de paz la ausencia de violencia estructural y la presencia de justicia social (paz positiva) (1969, pp. 167-192). Mientras que los primeros pretendían evitar que la expansión de la agenda disgregase sus propósitos originales, los segundos aspiraban a que graves injusticias no quedasen ocultas simplemente por la inexistencia de guerras. Aunque esta disputa no se resolvió formalmente, la mayoría de los investigadores para la paz han terminado aceptando la ampliación de la agenda, lo que ha tenido como consecuencia directa la percepción de que el trabajo de la Investigación para la Paz resulta expansivo e inacabado.

\section{B. La necesidad de una respuesta interdisciplinar}

La complejidad del objeto de estudio de la Investigación para la Paz ha obligado a la búsqueda de respuestas interdisciplinares para afrontar los desafíos que plantean la paz y la violencia (Alger, 1996, p. 2007). Como señalan los profesores Francisco Muñoz y Javier Rodríguez Alcázar, ${ }^{3}$ en la base de dicha complejidad se encuentra la propia naturaleza del conflicto, de carácter multicasual, multidimensional, que hace que cualquier reflexión en torno a la paz o a la violencia deba ir acompañada por una aproximación multidisciplinar (Muñoz \& Rodríguez Alcázar, 2000, p. 37).

La complejidad y la pluralidad temática que ofrece no solo el estudio de la paz, sino también de la violencia y de los conflictos, permite que puedan ser abordados desde variados enfoques y puntos de vista. Esto ha posibilitado que investigadores procedentes de diferentes disciplinas científicas hayan enriquecido la perspectiva general de la Investigación para la Paz con aportaciones teóricas, metodológicas y epistemológicas procedentes de otras áreas; combinando al menos tres enfoques generales: un enfoque práctico para la resolución de problemas basado en las necesidades, un enfoque racional cuantitativo y empírico-comparativo, y un enfoque estructuralista teórico (Roger \& Ramsbotham, 1999, p. 31). Entre la amplia base disciplinar de la Investigación para la $\mathrm{Paz}$ podemos citar las contribuciones de las diferentes Ciencias Sociales (como Ciencia Política, Sociología, Psicología, Antropología, Economía o Derecho), de las Humanidades (Historia, Geografía, Religión, Filosofía, Literatura, Lingüística, Artes) y de las Ciencias Naturales y Físicas (Física, Biología, Química, Matemáticas) (Stephenson, 1999, p. 810; Jeong, 1999).

3 Los profesores Francisco Muñoz y Javier Rodríguez Alcázar son miembros del Instituto de la Paz y los Conflictos de la Universidad de Granada, un centro de investigación que puede servir como modelo para ilustrar el carácter interdisciplinar de la Investigación para la Paz, ya que reúne en su seno a científicos procedentes de disciplinas tan dispares como Historia, Medicina, Química, Filosofía, Matemáticas o diferentes filologías. 


\section{Cuadro 2.1 La Investigación para la Paz, una respuesta multidisciplinar}

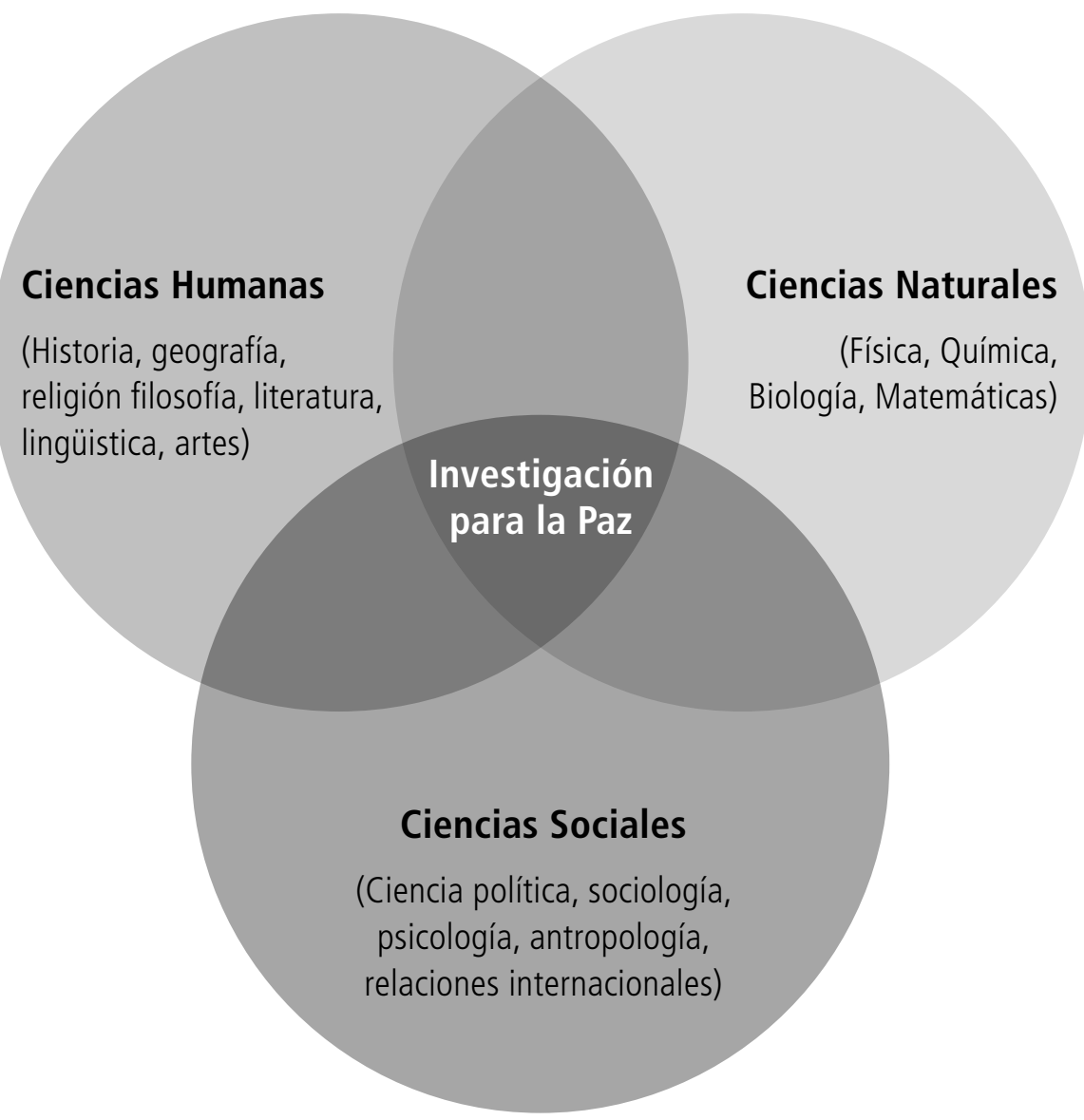

Fuente: Elaboración propia.

La práctica de la interdisciplinariedad ha llevado a la creación de espacios concretos donde los investigadores portadores de los conocimientos generados en sus particulares disciplinas los han podido compartir. Así han surgido redes y asociaciones de investigación para la paz como la International Peace Research Association (IPRA) o el Consortium for Peace Research, Education and Development (Copred); centros de estudios e investigación como el Peace Research Institute Oslo (PRIO) o el Stockholm International Peace Research Institute (Sipri); espacios de intercambio científico como los congresos de IPRA o los de la International Studies Association (ISA); y publicaciones periódicas como Journal of Conflict Resolution o Journal of Peace Research. De esta manera, los procesos de asociación de investigadores y académicos generan dinámicas que facilitan el debate, consolidan los consensos y originan grupos de opinión en torno a la paz y los conflictos. 
El trabajo conjunto, compartido, de todas esas disciplinas hace que los estudios sobre la paz y los conflictos tengan una personalidad globalizadora y que no queden estancados, por ejemplo, en un análisis puramente politicista, historicista o economicista. Tal y como señala Johan Galtung, la evolución lógica de esa interdisciplinariedad debe guiar a una transdisciplinaridad que suponga la integración de las perspectivas y metodologías de varias disciplinas para que la Investigación para la Paz sea más holística y global (Galtung, 1985, p. 144).

\section{Gestión pacífica y no violenta de los conflictos}

Otro elemento de esta disciplina es la pretensión por el fomento de la capacidad transformadora del ser humano frente a los conflictos del mundo contemporáneo, con la intención de elaborar metodologías de acción que posibiliten la gestión no violenta de los conflictos, previniendo el recurso a la utilización de la violencia y potenciando aquellas estrategias dirigidas a la construcción de paz (Curle, 1978; Muñoz \& Rodríguez Alcázar, 2004; Mitchell, 2005). Desde esta perspectiva, los conflictos son situaciones inherentes a las sociedades o grupos humanos que se relacionan entre sí y es el modo en el que se abordan lo que puede generar satisfacción o insatisfacción, sufrimiento o bienestar, equidad o inequidad, etc., lo que la ha llevado a apostar decididamente por su gestión pacífica y no violenta, dado los costes que genera la utilización de la violencia (Fisas, 1998, pp. 62-64).

Debido a esto, se han realizado estudios comparativos de procesos pacíficos y no pacíficos de cambio político y social, análisis de formas de prevención de los estallidos de la violencia o de su mitigación una vez aparecida, y exploraciones de los mecanismos necesarios para la reconciliación de sociedades divididas por la violencia. Ante esta situación podemos distinguir tres grandes enfoques para la gestión pacífica de los conflictos: la regulación de conflictos, la resolución de conflictos y la transformación de conflictos (Burgess \& Burgess, 2003; Deutsch, 1973; Fisher \& Ury, 1981; Reimann, 2004; Wallensteen, 2002), cada uno de los cuales se caracteriza por sostener sus propias concepciones sobre la paz, por utilizar metodologías de intervención diferentes y por el protagonismo de distintos actores (Checa Hidalgo \& Ghica, 2007).

\section{El análisis multinivel de los conflictos}

El interés de la Investigación para la Paz por estudiar los conflictos también la ha llevado al desarrollo de modelos de análisis para localizar las fuentes primarias de los conflictos contemporáneos. Tradicionalmente, existía una dicotomía institucionalizada entre aquellos estudios centrados en las dimensiones internas y los que estaban dirigidos a las dimensiones externas, lo que se ha considerado inadecuado para el correcto análisis de los conflictos. Para superar esta dicotomía, la Investigación para la Paz es partidaria de la utilización de un análisis multinivel, que sea capaz de generar una explicación más completa de los conflictos. En este sentido podemos citar el marco interpretativo para el análisis de conflictos elaborado por los profesores Oliver Ramsbotham, Tom Woodhouse y Hugh Miall para la identificación de las fuentes de los conflictos contemporáneos que se expone en la Tabla 1.

Como se puede apreciar, este marco interpretativo distingue entre el nivel global, el regional, el estatal, el grupal y el individual. De esta manera, la interpretación multinivel facilita la disección de los conflictos, permite identificar más fácilmente sus elementos fundamentales y otorga el análisis en profundidad de aquellos procesos que cortan transversalmente diferentes niveles. 


\section{Tabla 1. Marco de análisis de conflictos}

\begin{tabular}{|c|c|c|c|}
\hline & NIVEL & & EJEMPLO \\
\hline 1 & Global & \multicolumn{2}{|c|}{$\begin{array}{l}\text { Transición geopolítica, división económica Norte-Sur, restricciones medioambientales, } \\
\text { proliferación armamentística, disputas ideológicas. }\end{array}$} \\
\hline 2 & Regional & \multicolumn{2}{|c|}{ Patrones de clientelazgo, difusión, intervención, diásporas, movimientos de población. } \\
\hline \multirow{3}{*}{3} & \multirow{3}{*}{ Estatal } & Social & Sociedad débil: divisiones culturales, desequilibrio étnico. \\
\hline & & Económico & Economía débil: base de recursos pobre, pobreza relativa. \\
\hline & & Político & Organización política débil: gobierno partidista, ilegitimidad del régimen. \\
\hline 4 & Grupal & \multicolumn{2}{|c|}{ Movilización de grupo, dinámicas intergrupales. } \\
\hline 5 & Elite / Individual & \multicolumn{2}{|c|}{ Políticas de exclusión, intereses de las facciones, liderazgo depredador. } \\
\hline
\end{tabular}

Fuente: Ramsbotham et al., 2005, p. 97.

\section{E. El enfoque global y multicultural}

La adopción de una perspectiva global y holística para el análisis de sus objetos de estudio es otro de los elementos esenciales de la Investigación para la Paz. Esto se debe a que en su visión de los fenómenos políticos, sociales, económicos o ecológicos, así como de las problemáticas detectadas en cada uno de estos ámbitos, se aprecia una interrelación a nivel mundial cada vez mayor (Muñoz \& Rodríguez Alcázar, 2000, p. 39). Aunque una perspectiva global no debe ser un obstáculo para identificar dinámicas locales, sectoriales o grupales, la globalización ha hecho necesaria la construcción de una agenda mundial de la paz que no se limite a los problemas a escala micro o meso.
Este enfoque ha tenido como consecuencia positiva el cuestionamiento de la tradicional perspectiva eurocéntrica y la profundización de la reflexión sobre la manera de percibir e interpretar los fenómenos sociales, pero también sobre la forma de comunicar y de actuar. Sin embargo, obliga a un importante esfuerzo, ya que requiere el tener en cuenta las aportaciones tanto de otras disciplinas, como de otras culturas, con los objetivos de conseguir un mejor entendimiento de la realidad y de elaborar mecanismos de intervención eficaces. Aunque la adopción de una perspectiva global es una aspiración ampliamente reconocida hoy en día, la cuestión de la validez multicultural ha sido ampliamente cuestionada y su evolución hacia la transcultu- 
ralidad se presenta como un importante desafío para los modelos de gestión de conflictos (Roger \& Ramsbotham, 1999, pp. 32-33).

\section{F. El análisis objetivo y el compromiso normativo}

Esta disciplina aúna en su seno tanto tareas analíticas como un fuerte compromiso normativo. Por ello, busca el reconocimiento de aquellas experiencias humanas donde los conflictos y los cambios sociales y políticos se han gestionado de manera pacífica y no violenta, qué circunstancias lo han posibilitado, y la manera en la que pueden ser reproducidos en otros contextos.

Si bien sus orígenes se fundamentaron en la investigación cuantitativa y en el estudio empírico comparativo, la mayoría de los especialistas que se acercaron a ella lo hicieron atraídos por sus preocupaciones y compromisos de carácter ético (Roger \& Ramsbotham, 1999, p. 14). Así, su construcción se ha producido sobre un explícito compromiso por la paz como un valor en sí mismo (Stephenson, 1999, p. 810).

Con estos planteamientos, podemos afirmar, siguiendo las palabras de Johan Galtung, que la Investigación para la Paz se ha construido sobre tres bases: la investigación empírica, la investigación crítica y la investigación constructiva (1996). De este modo, se ha producido la sistemática comparación de teorías con la realidad, revisando las teorías si no coinciden con los datos; se ha comparado la realidad con los valores, intentando cambiarla si no coincide con éstos; y se han intentado ajustar las teorías a los valores tratando de producir nuevas realidades.

En ese sentido, tal y como resaltan los profesores Vicent Martínez Guzmán y Francisco Muñoz, la paz se ha convertido en una categoría normativa que pretende indicar cómo se deben gestionar los conflictos para satisfacer las necesidades y aspiraciones de los individuos y de las sociedades de la manera más satisfactoria y justa posible (Martínez Guzmán \& Muñoz, 2004, p. 597).

\section{G. La estrecha relación entre teoría y práctica}

El último elemento característico de la Investigación para la Paz es la estrecha relación entre teoría y práctica, otorgando a esta disciplina el apelativo de ciencia aplicada, cuya clara intencionalidad es la orientación de la acción política. Así, como señala Carolyn Stephenson, sus construcciones teóricas pretenden afectar los ámbitos de decisión política, en un intento de avanzar en la realización de esas propuestas de nuevas realidades que se han mencionado anteriormente (1999, p. 810).

De este modo, y utilizando la comparación que ha realizado Johan Galtung entre las Ciencias de la Salud y la Investigación para la Paz, podemos contemplar en esta última los procesos de diagnóstico-pronóstico-terapia, donde el diagnóstico es un análisis basado en los datos; el pronóstico consiste en predicciones basadas en la teoría sobre un contexto determinado; y la terapia supone una intervención basada en valores y teorías (Galtung, 1996).

\section{Etapas de desarrollo de la Investigación para la Paz}

Si repasamos algunos de los trabajos que han tratado de sistematizar las fases que ha atravesado la Investigación para la Paz a lo largo de su evolución, resulta evidente que la historia de esta disciplina atraviesa principalmente cuatro fases: los orígenes (1914-1945), la institucionalización (1945-1970), la expansión (1970-1990), y la revisión y consolidación (desde 1990) (Harty \& Modell, 1991; Kriesberg, 1997; Harto de Vera, 2004; Ramsbotham et al., 2005).

La historia de la investigación empírica e incluso cuantitativa sobre la guerra y la paz se puede rastrear hasta comienzos del siglo XIX, cuando se documentan diferentes intentos para estudiar las pérdidas de vidas humanas causadas por la guerra, para cuantificar el número de batallas y guerras o para estimar los gastos militares (Wiberg, 1988, p. 32). Sin embargo, los primeros 
intentos de enfrentarse desde una perspectiva científica (en sentido positivista) a la problemática de la paz, datan de los años posteriores a la Primera Guerra Mundial, en el periodo de entreguerras, cuando activistas, intelectuales y políticos tomaron conciencia de la necesidad de reaccionar ante la barbarie (Cortright, 2008). De esos años datan los trabajos de Pitirim Sorokin que estudian estadísticamente el fenómeno de la guerra, desde el siglo VI antes de Cristo hasta el siglo XX; de Lewis F. Richardson que analizó las causas de la guerra y creó un modelo matemático aplicado al rearme; y de Quincy Wright que publicó un texto sobre la guerra con un estudio ya multidisciplinar del tema.

El desarrollo de los estudios sobre la paz, la guerra y los conflictos se produjo a partir del final de la Segunda Guerra Mundial, cuando la aparición de las armas nucleares supuso una amenaza exponencial para la humanidad. Así, el proceso de institucionalización de la Investigación para la Paz se produjo en un contexto internacional definido por el enfrentamiento entre las dos superpotencias (Estados Unidos y la Unión Soviética) que emergieron del conflicto y que se denominó Guerra Fría. En esta fase se fundaron y establecieron las primeras instituciones que iban a dedicarse a la investigación, como el Peace Research Laboratory de St. Louis (EE.UU.) en 1945 (Harto de Vera, 2004, p. 47).

Este proceso de institucionalización tuvo dos focos fundamentales, uno en Michigan, EE.UU, que agrupó a una corriente pragmática y minimalista, y otro en los países escandinavos, concretamente en Noruega y Suecia, de tendencia estructuralista y maximalista (Ramsbotham et al., 2005, pp. 39-47). En 1957, Kenneth Boulding junto a Anatol Rapoport crearon la primera revista especializada, el Journal of Conflict Resolution en la Universidad de Michigan, y, dos años más tarde, se fundó en la misma universidad el Center for Research on Conflict Resolution. En torno a ambas iniciativas, un grupo de investigadores comenzaron a trabajar en la construcción de una teoría científica acerca del conflicto y centraron sus estudios en la temática de la prevención de la guerra. Incluso acuñaron el término resolución de conflictos, tratando de evidenciar su intención de abordar el objeto de estudio desde una perspectiva puramente técnica y de alejarse de las connotaciones políticas de izquierdas que podía acarrear la utilización del término investigación para la paz. (Harty \& Modell, 1991).

Mientras tanto, en Europa emergió otro foco de investigación, pero con una agenda más amplia, con la figura de Johan Galtung como su más significativo impulsor, quien en 1960 ayudó a crear una unidad para la investigación de los conflictos y de la paz en el Institute for Social Research de la Universidad de Oslo, el antecesor del PRIO, y en 1964 fundó la revista Journal of Peace Research (Ramsbotham et al., 2005, pp. 41-42). Su influencia hizo que la Investigación para la Paz fuese más allá de la prevención de la guerra y estudiara también las condiciones para el establecimiento de relaciones pacíficas entre las partes en conflicto y la búsqueda de una paz positiva, mediante la erradicación de aquellas estructuras políticas y socioeconómicas que sostenían la violencia.

Poco a poco se crearon nuevos centros de investigación como el Polemological Institute, en Holanda, o el SIPRI, en Suecia; y surgió IPRA como un intento para coordinar los esfuerzos de la Investigación para la Paz a nivel internacional, cuyo primer congreso se realizó en 1965, bajo la organización de John Burton, otro de los padres fundadores de esta disciplina. Burton se centró en el análisis del conflicto y avanzó en la tesis de la consideración del conflicto como parte de la naturaleza humana (Burton, 1990).

A comienzos de los años setenta, en un contexto internacional donde la tensión entre las superpotencias se había reducido, la Investigación para la Paz, apoyándose en una extensa gama de disciplinas y con una razonable base institucional, había ampliado su objeto de estudio desde la gran preocupación de la década de 1950, cómo evitar la guerra nuclear, a nuevas áreas de interés como la eliminación de las desigualdades y las injusticias en el sistema global y la consecución de un equilibrio ecológico. 
Los años siguientes, tal y como señalan Oliver Ramsbotham, Tom Woodhouse y Hugh Miall, asistieron a diferentes intentos por formular comprensiones teóricas de los conflictos destructivos a tres niveles. Primero, a nivel interestatal, el principal esfuerzo fue convertir la disuasión entre las superpotencias en acuerdos formales beneficiosos para ambos actores; después, a nivel estatal, el trabajo se centró en el desarrollo de la mediación familiar, laboral y comunitaria, así como de la resolución alternativa de disputas; y en tercer lugar, entre el nivel interestatal y el estatal, surgió la definición, el análisis y la prescripción sobre los llamados conflictos de raíces profundas, conflictos intratables o conflictos sociales prolongados (Ramsbotham et al., 2005, pp. 47-48).

Durante este periodo, su expansión fue muy importante, abriéndose nuevos campos para la aplicación de las prácticas de resolución de conflictos, propagándose las ideas a través de distintas revistas especializadas; publicándose numerosos estudios de casos en los que se aplicaban las técnicas desarrolladas a nivel teórico por esta disciplina, particularmente en torno a la mediación en conflictos; e incorporando a los programas de formación de instituciones académicas y no académicas el aprendizaje de la negociación y la mediación (Kriesberg, 1997, p. 58).

Resulta más difícil evaluar a la Investigación para la Paz después del fin de la Guerra Fría, ya que aunque hay quienes argumentan que es una disciplina que sigue creciendo, otros afirman que es un campo en declive tanto en número de participantes y programas como por su fraccionamiento intelectual. Lo cierto es que es posible documentar ambas argumentaciones debido a que, mientras que el núcleo de estos estudios está definido, las fronteras permanecen flexibles, lo que hace que la disciplina se caracterice por una enorme diversidad y por su estrecha relación con otros campos de estudio como la Ciencia Política, la Sociología o las Relaciones Internacionales (Stephenson, 1999, pp. 818819). Lo que sí parece indiscutible es que a lo largo de los años noventa se ha producido su consolidación definitiva.
De esta forma, el cambio del contexto internacional en la década del noventa motivó que la Investigación para la Paz tuviese que modificar sus planteamientos y se iniciase una revisión teórica de la disciplina para adaptarse al nuevo contexto, puesto que a pesar de los anuncios que presumían del Fin de la Historia del conflicto (Fukuyama, 1992), pronto se hizo evidente la necesidad de que esta disciplina abordara las nuevas formas que adoptaban la violencia y los conflictos.

En este proceso de adaptación teórica a las nuevas realidades han surgido nuevas perspectivas en la estrategia de construcción de la paz, consolidando el paradigma de la transformación de conflictos desde abajo, impulsado particularmente por John Paul Lederach (Vinyamata, 2005). Esto supone un enfoque a largo plazo que tiene en cuenta tanto la dimensión estructural como la relacional y cultural, poniendo énfasis en los cambios que habrán de producirse en los individuos, en el sistema de relaciones, en las culturas y en los países a partir de su propia experiencia de superación de los conflictos violentos, del que son claramente deudoras las intervenciones internacionales noviolentas (Lederach, 1995).

Siguiendo esta misma línea, debemos mencionar también las aportaciones realizadas por la Teoría Social Crítica, cuyas proposiciones sostienen que mientras que la perspectiva tradicional para la resolución o la gestión de conflictos puede llevar a reforzar y perpetuar las instituciones dominantes y generadoras de violencia, sin abordar las causas profundas de los conflictos; la Teoría Crítica trata de analizar las estructuras que sostienen los conflictos para ofrecer alternativas que terminen con los órdenes injustos que generan violencia (Hoffman, 1987; Nordstrom, 1995; Jabri, 1996).

A ambos desarrollos debemos añadir que el análisis crítico desde la perspectiva de género ha denunciado la invisibilización de las mujeres como víctimas de la violencia y ha resaltado su papel en la creación de modelos de regulación pacífica de conflictos especialmente 
a nivel local (Duffey, 1998). Apoyándose en sus experiencias, este enfoque ha abogado por una mayor presencia de la mujer en las instancias que diseñen las instituciones que regularán la convivencia futura, para evitar la reproducción de las estructuras y discursos discriminatorios que generan violencia, fruto de lo cual han surgido iniciativas como la Resolución $1325 .^{4}$

Finalmente, y tal y como plantean Oliver Ramsbotham, Tom Woodhouse y Hugh Miall, a los anteriores enfoques hay que sumar uno más, el ofrecido desde la revisión de la cuestión cultu$\mathrm{ral}$, que se cuestiona hasta qué punto las actividades para la resolución de conflictos pueden ser tomadas como actividades universalmente válidas para cualquier contexto cultural, puesto que incorporan determinados presupuestos propios de la cultura occidental para los que no existe consenso cuando nos encontramos en escenarios y contextos culturales no occidentales (Ramsbotham et al., 2005, pp. 305-310). Si bien hay autores para los que la variación cultural no es relevante para la gestión de conflictos (Burton, 1990; Zartman, 1997), o cuya consideración se reduce al hecho de percibirla como una más (Bercovitch, 1996), existe una nueva perspectiva que le otorga a la variación cultural una importancia fundamental (Avruch \& Black, 1991; Lederach \& Wehr, 1991; Lederach 1995; 1997; Galtung, 1990; 1996). Este nuevo enfoque enfatiza la importancia de la comprensión de las prácticas indigenas de gestión de conflictos y construcción de paz para que su fortalecimiento, mediante, por ejemplo, intervenciones internacionales noviolentas, permita la apropiación local de la transformación de los conflictos.

De esta manera, a partir de la suma de todos estos enfoques, poco a poco se ha contribuido a la mejora de la comprensión de los fenómenos relacionados con los conflictos, la paz y la violencia, enriqueciendo la disciplina de la Investigación para la Paz y contribuyendo a su consolidación definitiva en el mundo académico, tal y como se puede constatar a partir de los trabajos de carácter enciclopédico que reúnen los distintos saberes de esta ciencia (Burgess \& Burgess, 1997; Kurtz, 1999; López Martínez, 2004; Young, 2010).

\section{La agenda de la Investigación para la Paz}

Desde los inicios hasta la actualidad, la Investigación para la Paz ha ido ampliando su radio de acción de manera paulatina. A grandes rasgos y siguiendo la clasificación realizada por el profesor Hakan Wiberg, la agenda de la Investigación para la Paz puede dividirse en tres etapas. En la primera encontramos una vieja agenda, que durará hasta finales de los años sesenta, centrada por un lado en el análisis de las causas de la guerra y de los medios para la resolución de los conflictos y, por otro lado, en el estudio de los temas relativos a la carrera armamentística, al control de armas y al desarme. La segunda etapa, iniciada en la década de los setenta, vio cómo se añadían nuevos temas a la disciplina dando origen a una nueva agenda que incluyó los trabajos sobre las relaciones de dominación y dependencia, y sobre el desarrollo. Finalmente, el final de la Guerra Fría marcó el inicio de la tercera etapa donde se creó una post nueva agenda, que incorporó nuevos temas que se sumaron a los de la vieja y la nueva agenda, tales como la comprensión de las identidades y del nacionalismo o el análisis de los procesos migratorios mundiales (Wiberg, 1993).

Hay que tener presente, como ya hemos indicado, que la Investigación para la Paz estuvo marcada en sus inicios por la experiencia de las guerras mundiales, lo que determinó que su

4 La Resolución 1325 del Consejo de Seguridad de Naciones Unidas, adoptada en el año 2000, abordaba por primera vez en el seno de esta institución la situación de las mujeres en los conflictos bélicos y en ella se exhortaba a actuar para lograr una mayor inclusión de las mujeres en los procesos de construcción de la paz y de reconstrucción postconflicto. 
atención prioritaria se dirigiese en sus primeros momentos hacia la prevención de la guerra, mediante el estudio de sus causas, y hacia el armamentismo, especialmente centrada en las armas nucleares. En este contexto, se realizaron estudios empíricos sobre la magnitud y evolución de los arsenales, sobre las fuentes de tensión geoestratégica.

Será Johan Galtung quien impulse la ampliación de la agenda a partir de los años setenta al criticar la agenda minimalista. Distinguiendo entre los conceptos de paz negativa y paz positiva, Galtung afirmaba que el estado de paz exigía no solamente la ausencia de una violencia abierta, sino también de la violencia estructural en forma de explotación económica y social, ya que existían situaciones donde no se producían guerras o violencia directa, pero donde estaban vigentes regímenes autoritarios o profundamente injustos que causaban sufrimiento (Galtung, 1969). Esto significaba que hasta finales de la década de los sesenta, la Investigación para la Paz se había centrado en el estudio de la paz negativa, fundamentada en la prevención o erradicación de la violencia directa, especialmente las guerras. Dicha violencia directa era definida por el propio Galtung como: "el tipo de violencia donde hay un actor que comete la violencia como personal o directa", para distinguirla de aquella violencia estructural o indirecta que se produce sin la presencia de dicho actor (1969, p. 170). Debido a esa desatención que había detectado, propuso situar también el objeto de estudio en el análisis crítico de las estructuras y de los posibles esfuerzos necesarios para transformar las estructuras violentas, con la intención de alcanzar una pazpositiva construida sobre ideas como armonía, cooperación e integración entre los grupos humanos, entendiendo que el papel de la Investigación para la Paz debía considerar tanto los aspectos negativos como los positivos de la paz (Galtung, 1985).

Este enfoque fue finalmente asumido por la mayoría de los investigadores para la paz y llevó a la expansión de la agenda de esta disci- plina, que añadió a sus preocupaciones tradicionales otras nuevas como las desigualdades globales Norte-Sur o los problemas medioambientales (Homer-Dixon, 1994; Gleditsch, 1998). La tradicional atención a la amenaza nuclear se extendió también a las armas químicas y biológicas, manteniéndose el interés por el control de armas y el desarme, y el análisis de los peligros inherentes de posturas disuasorias aliadas se amplió a las estrategias de defensa mutua. Otra área que experimentó un fuerte crecimiento fue el estudio empírico de los procesos de negociación y de mediación, y el análisis de los conflictos sociales prolongados (Rogers \& Ramsbotham, 1999, pp. 23-25).

La extensión del campo de acción de la Investigación para la Paz no ha dejado de ampliarse y a partir de la década de los noventa pueden encontrarse en su nueva agenda, tal y como afirma Ho-Won Jeong, una serie de cuestiones que caracterizan la transición estructural de la política mundial del siglo XX al siglo XXI, como son el interés por la globalización, la formación de las identidades, los requerimientos para la satisfacción de las necesidades básicas, los derechos humanos, el desarrollo regional o la actuación de la sociedad civil (Jeong, 1999). Sin embargo, como señala el profesor Luc Reychler (2006), algunos de los mayores retos de la disciplina continuarán siendo los altos niveles manifiestos y potenciales de violencia existentes y la naturaleza predominantemente reactiva de los esfuerzos por su prevención.

Llegados a este punto puede ser útil realizar un resumen de los temas contenidos en la agenda de la Investigación para la Paz y, para ello, una buena opción es utilizar las áreas de trabajo de la International Peace Research Association (IPRA), es decir, las áreas en las que los investigadores se agrupan para compartir sus investigaciones y para discutir su agenda de interés, que, tal y como se muestran en la Tabla 2, ilustran de manera representativa cuales son las temáticas principales de la disciplina. 
Tabla 2. Áreas de trabajo de IPRA

\begin{tabular}{|c|}
\hline COMISIONES DE TRABAJO \\
\hline Arte y Paz \\
\hline Conflictos Internos \\
\hline Cultura de Paz y Comunicaciones \\
\hline Derechos de los Pueblos Indígenas \\
\hline Derechos Humanos Internacionales \\
\hline Ecología y Paz \\
\hline Economía Política Global \\
\hline Educación para la Paz \\
\hline Europa Oriental \\
\hline Género y Paz \\
\hline Historia de la Paz \\
\hline Juventud y Paz \\
\hline Migraciones Forzosas \\
\hline Movimientos de Paz \\
\hline Noviolencia \\
\hline Periodismo de Paz \\
\hline Reconciliación \\
\hline Religión y Paz \\
\hline Resolución de Conflictos y Peacebuilding \\
\hline Seguridad y Desarme \\
\hline Teorías de Paz \\
\hline GRUPOS DE TRABAJO \\
\hline Conocimiento y Paz \\
\hline Deporte y Paz \\
\hline Desarrollo y Paz \\
\hline Estatuto de la Tierra \\
\hline Evaluación de las Actividades de Desarrollo y Paz \\
\hline Gobernanza Mundial y Paz \\
\hline Negociaciones de Paz y Mediación \\
\hline Oriente Medio \\
\hline Psicología de la Paz \\
\hline
\end{tabular}

\section{Reflexiones finales}

Antes de finalizar este artículo es necesario realizar tres precisiones. En primer lugar, es preciso considerar que la evolución y expansión del campo de acción de la Investigación para la Paz también refleja las preocupaciones existentes en el seno de la sociedad internacional, donde poco a poco sus miembros han prestado una mayor atención hacia los análisis y resultados de esa disciplina, y han ido incorporando algunos de sus enfoques y de sus recomendaciones, tal y como se pone de manifiesto en la Tabla 3. Buena muestra de esta tendencia es la Declaración Sobre una Cultura de Paz, realizada por la Asamblea General de la Unesco en 1999 (Naciones Unidas, 1999).

En segundo lugar, no debemos olvidar que, aunque los investigadores para la paz tienen hoy una función más compleja que en los tiempos de la Guerra Fría, tal y como afirmaba el profesor Peter Wallensteen al referirse a los orígenes de la disciplina, siguen siendo espoleados por la continua existencia de conflictos violentos y por las aspiraciones del idealismo en sus estudios teóricos y empíricos para la búsqueda de nuevas explicaciones con la intención de interpretar mejor la realidad, pasada y presente, y realizar propuestas para la construcción de un mundo más justo y pacífico (Wallensteen, 1988, pp. 26).

Finalmente, en tercer lugar, también hay que señalar que el creciente proceso de especialización que se ha producido en el seno de la Investigación para la Paz es un fenómeno que da buena muestra de la amplitud, de la expansión y del interés que genera este nuevo campo de estudio. Esta dinámica, fruto de su carácter inter y transdisciplinar, que tiende también a la fragmentación, representa un importante reto para la evolución de la disciplina en los próximos años.

Fuente: IPRA, Congreso Mundial 2008. 
Tabla 3. Emergencia de herramientas de paz en la sociedad internacional

\begin{tabular}{|c|c|c|c|c|c|c|}
\hline & Siglo XIX & $\begin{array}{c}\text { Liga de las } \\
\text { Naciones (1919) }\end{array}$ & $\begin{array}{l}\text { Carta de la } \\
\text { ONU (1945) }\end{array}$ & $\begin{array}{l}\text { Práctica ONU } \\
(1950-1989)\end{array}$ & $\begin{array}{l}\text { Práctica ONU } \\
(1990-)\end{array}$ & $\begin{array}{l}\text { Sociedad } \\
\text { Civil }\end{array}$ \\
\hline \multirow{8}{*}{ 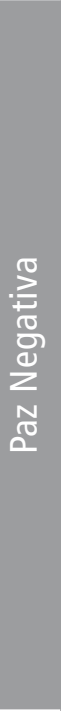 } & Diplomacia & & & & & \\
\hline & $\begin{array}{l}\text { Equilibrio } \\
\text { de Poder }\end{array}$ & & & & & \\
\hline & & $\begin{array}{l}\text { Seguridad } \\
\text { colectiva }\end{array}$ & Seguridad colectiva & Seguridad colectiva & Seguridad colectiva & \\
\hline & & Arreglo pacífico & Arreglo pacífico & Arreglo pacífico & Arreglo pacífico & \\
\hline & & & & Peacekeeping & Peacekeeping & $\begin{array}{l}\text { Diplomacia } \\
\text { de nivel II }\end{array}$ \\
\hline & & $\begin{array}{l}\text { Control de armas } \\
\text { / desarme }\end{array}$ & $\begin{array}{l}\text { Control de armas / } \\
\text { desarme }\end{array}$ & $\begin{array}{l}\text { Control de armas / } \\
\text { desarme }\end{array}$ & $\begin{array}{l}\text { Control de armas / } \\
\text { desarme }\end{array}$ & Conversión \\
\hline & & & & & $\begin{array}{l}\text { Intervención } \\
\text { humanitaria }\end{array}$ & $\begin{array}{l}\text { Defensa } \\
\text { defensiva }\end{array}$ \\
\hline & & & & & $\begin{array}{l}\text { Diplomacia } \\
\text { preventiva }\end{array}$ & Noviolencia \\
\hline \multirow{8}{*}{ 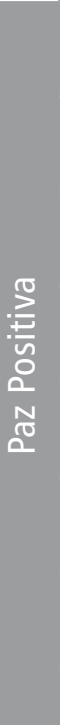 } & & & $\begin{array}{l}\text { Cooperación } \\
\text { funcionalista }\end{array}$ & $\begin{array}{l}\text { Cooperación } \\
\text { funcionalista }\end{array}$ & $\begin{array}{l}\text { Cooperación } \\
\text { funcionalista }\end{array}$ & $\begin{array}{l}\text { Diplomacia } \\
\text { ciudadana }\end{array}$ \\
\hline & & & Autodeterminación & Autodeterminación & Autodeterminación & $\begin{array}{l}\text { Auto- } \\
\text { confianza }\end{array}$ \\
\hline & & & Derechos Humanos & Derechos Humanos & Derechos Humanos & $\begin{array}{l}\text { Perspectiva } \\
\text { feminista }\end{array}$ \\
\hline & & & & $\begin{array}{l}\text { Desarrollo } \\
\text { económico }\end{array}$ & $\begin{array}{l}\text { Desarrollo } \\
\text { económico }\end{array}$ & $\begin{array}{l}\text { Educación } \\
\text { para la paz }\end{array}$ \\
\hline & & & & Equidad económica & $\begin{array}{l}\text { Equidad } \\
\text { económica }\end{array}$ & \\
\hline & & & & $\begin{array}{l}\text { Equidad } \\
\text { comunicativa }\end{array}$ & $\begin{array}{l}\text { Equidad } \\
\text { comunicativa }\end{array}$ & \\
\hline & & & & Equilibrio ecológico & $\begin{array}{l}\text { Equilibrio } \\
\text { ecológico }\end{array}$ & \\
\hline & & & & Gobernanza global & Gobernanza global & \\
\hline
\end{tabular}

Fuente: Adaptado de Alger, 2006, p. 10.

En resumen y para concluir este artículo de revisión, debemos señalar que la Investigación para la Paz cuenta en la actualidad con un conjunto de conocimientos, metodologías y actitudes que le permiten enfrentarse con garantías al estudio de la amplia gama de problemáticas existentes en la sociedad internacional, desde la violencia a los múltiples y variados conflictos y amenazas para la seguridad presentes a comien- zos del siglo XXI. Para ello, como ya se ha puesto de manifiesto y dado su carácter interdisciplinar, la Investigación para la Paz se basa y a la vez se superpone, a diversos campos de estudios, posee un enfoque holístico combinado con metodologías cuantitativas y empíricas, y tiene un declarado compromiso normativo con el análisis de las condiciones para el cambio social y político sin que tenga que ser usada la violencia. 


\section{Referencias bibliográficas}

Alger, Ch. (1996). Introduction: Reflections on Peace Research Traditions. International Journal of Peace Studies, 1(1), 1-4.

(2006). The United Nations System: A Reference Handbook. Santa Barbara: ABCCLIO.

. (2007). Peace studies as a transdiciplinary project. En Ch. Webel \& J. Galtung (Eds.), Handbook of Peace and Conflict Studies (pp. 299-318). Londres-New York: Routledge.

Avruch, K. \& Black, P. (1991). The culture question and conflict resolution. Peace and Change, Vol. 16, 1, 22-45.

Bercovitch, J. (Ed.). (1996). Resolving International Conflicts: The Theory and Practice of Mediation. Boulder: Lynne Rienner.

Boulding, K. (1977). Twelve friendly quarrels with Johan Galtung. Journal of Peace Research, 14(1), 77-86.

Burgess, H., \& Burgess, G. (1997). Encyclopedia of conflict resolution. Santa Barbara: ABC-CLIO.

Burgess, G. y Burgess, H. (2003). Beyond intractability. Boulder: Conflict Research Consortium, University of Colorado. Recuperado de www. beyondintractability.org

Burton, J. (1990). Conflict: Resolution and Provention. Londres: Macmillan.
Checa Hidalgo, D., \& Ghica, L. A. (2007). Gestionarea crizelor si a conflictelor internationale. En L. A. Ghica \& M. Zulean (Eds.), Politica de Securitate Nationala (pp. 205-245). Bucarest: Polirom.

Cortright, D. (2008). Peace. A bistory of movements and ideas. Cambridge: Cambridge University Press.

Curle, A. (1978). Conflictividad y pacificación. Barcelona: Herder.

Deutsch, M. (1973). The resolution of conflict: constructive and destructive processes. New Haven: Yale University Press.

Duffey, T. (1998). Culture, Conflict Resolution and Peacekeeping. An Analysis with Special Reference to the Operations in Somalia (Tesis doctoral). Bradford: Department of Peace Studies, University of Bradford.

Fisas, V. (1998). Cultura de pazy gestión de conflictos. Barcelona: Icaria.

Fisher, R., \& Ury, W. (1981). Getting to yes: How to negotiate without giving in. Londres: Arrow Books.

Fukuyama, F. (1992). El fin de la bistoria y el último hombre. Barcelona: Planeta.

Galtung, J. (1969). Violence, peace and peace research. Journal of Peace Research, 6(3), 167-192. 
(1985). Twenty-Five years of Peace

Research: Ten challenges and some responses. Journal of Peace Research, 22(2), 141-158.

. (1990). Cultural violence. Journal of Peace Research, 27(3), 291-305.

. (1996). Peace by Peaceful Means: Peace and Conflict, Development and Civilization. Oslo: PRIO.

Gleditsch, N. P. (1998). Armed conflict and the environment. A critique of the literature. Journal of Peace Research, 35(3), 381-400.

Harto de Vera, F. (2004). Investigación para la paz. $y$ resolución de conflictos. Valencia: Tirant lo Blanch.

Harty, M., \& Modell, J. (1991). The first Conflict Resolution Movement, 1956-1971: An attempt to institutionalize applied interdisciplinary social science. Journal of Conflict Resolution, 35(4), 720-758.

Hoffman, M. (1987). Critical theory and the inter-paradigm debate. Millennium: Journal of International Studies, 16(2), 234-262.

Homer-Dixon, T. F. (1994). Environmental scarcities and violent conflict: Evidence from Cases. International Security, 19(1), 5-40.

Jabri, V. (1996). Discourses on Violence: Conflict Analysis Reconsidered. Manchester: Manchester University Press.

Jeong, H. (Ed.). (1999). The new agenda for Peace Research. Aldershot: Ashgate.

Kriesberg, L. (1997). The development of the Conflict Resolution field. En W. Zartman \& L. Rasmussen (Eds.), Peacemaking in International Conflict. Methods \& Techniques (pp. 51-77). Washington: United States Institute of Peace Press.

Kurtz, L. (Ed.). (1999). Encyclopedia of violence, peace and conflict. San Diego: Academic Press.
Lederach, J. P. (1995). Preparing for peace: Conflict transformation across cultures. New York: Syracuse University Press.

Lederach, J. P. (1997). Building Peace: Sustainable Reconciliation in Divided Societies. Washington: United States Institute of Peace.

Lederach, J. P. \& Wehr, P. (1991). Mediating conflict in Central America. Journal of Peace Research, 28(1), 85-98.

López Martínez, M. (Ed.). (2004). Enciclopedia de Paz y Conflictos. Granada: Universidad de Granada.

Martínez Guzmán, V., \& Muñoz, F. A. (2004). Investigación para la paz. En M. López Martínez (Ed.), Enciclopedia de Paz y Conflictos (pp. 595-598). Granada: Universidad de Granada.

Mitchell, Ch. (2005). Conflict, social change and confict resolution: An inquiry. En D. Bloomfield, M. Fischer \& B. Schmelzle (Eds.), Berghof handbook for conflict transformation. Berghof Research Center for Constructive Conflict Management. Recuperado de www.berghof-handbook.net

Muñoz, F. A., \& Rodríguez Alcázar, F. J. (2000). Una agenda de la Investigación para la Paz. En F. J. Rodríguez Alcázar (Ed.), Cultivar la paz: Perspectivas desde la Universidad de Granada (pp. 27-51). Granada: Universidad de Granada. . (2004). Agendas de la Paz. En B. Molina Rueda \& F. A. Muñoz (Eds.), Manual de Pazy Conflictos (pp. 426-444). Granada: Universidad de Granada.

Naciones Unidas. (1999). Declaración y Programa de Acción sobre una cultura de par. Asamblea General, documento A/53/243, 6 de Octubre. Recuperado de http://www.unesco.org/cpp/ uk/declarations/2000.htm

Nordstrom, C. (1995). Contested identities, essentially contested powers. En K. Rupesinghe 
(Ed.), Conflict Transformation (pp. 93-111). Londres: Macmillan.

Ramsbotham, O., Miall, H., \& Woodhouse, T. (2005). Contemporary conflict resolution. Cambridge-Malden: Polity Press.

Reimann, C. (2004). Assessing the state-of-the-art in conflict transformation: reflections from a theoretical perspective. En D. Bloomfield, M. Fischer \& B. Schmelzle (Eds.), Berghof handbook for confict transformation. Berghof Research Center for Constructive Conflict Management. Recuperado de www.berghof-handbook.net

Reychler, L. (2006). Challenges of Peace Research. International Journal of Peace Studies, 11(1), 1-16.

Rogers, P., \& Ramsbotham, O. (1999). Then and now: Peace Research, past and future. Political Studies, 47, 740-754.

Stephenson, C. M. (1999). Peace Studies, Overview. En L. Kurtz (Ed.), Encyclopedia of violence, peace and conflict. Vol. 2 (pp. 809-820). San Diego: Academic Press.

Vinyamata, E. (2005). Conflictología. Curso de Resolución de Conflictos. Barcelona: Ariel.
Wallensteen, P. (1988). The origins of Peace Research. En P. Wallensteen (Ed.), Peace Research. Achievements and challenges (pp. 7-29). BoulderLondres: Westview Press.

Wallensteen, P. (2002). Understanding conflict resolution: War, peace and the global system. Londres: Sage.

Wiberg, H. (1988). The peace research movement. En P. Wallensteen (Ed.). Peace Research. Achievements and challenges (pp. 30-53). Boulder-Londres: Westview Press.

Wiberg, H. (1993). European peace research in the 1990s. En J. Balázs \& H. Wiberg (Eds.), Peace Research for the 1990s (pp. 9-25). Budapest: Akadémiai Kiadó.

Young, N. (Ed.). (2010). The Oxford International Encyclopedia of Peace. Oxford-New York: Oxford University Press.

Zartman, W. (1997). Toward the resolution of international conflicts. En W. Zartman \& J. L. Rasmussen (Eds.), Peacemaking in International Conflict: Methods and Techniques (pp. 3-22). Washington: 